\title{
Cross-Modal Associations between Color and Haptics
}

\author{
Nadiya Slobodenyuk • Yasmina Jraissati • Ali Kanso • \\ Lama Ghanem • Imad Elhajj
}

Published online: 4 March 2015

(C) The Psychonomic Society, Inc. 2015

\begin{abstract}
The objective of the present study was to explore cross-modal associations between color and tactile sensation while using haptically rendered virtual stimuli with substance properties of roughness/smoothness, hardness/softness, heaviness/lightness, elasticity/inelasticity, and adhesiveness/ nonadhesiveness. The stimuli with the indicated properties were rendered with the aid of SensAble PHANTOM $\mathrm{OMNI}^{\circledR}$ haptic device. The experimental setup required the participants to use exploratory procedures typical to real object interaction, and select a color from the HSV color space that matched the experienced sensation. The findings of our investigation reveal systematic mapping between color characteristics and intensity of the haptic stimuli. Qualitatively different haptic sensations, however, produced relatively similar patterns of cross-modal associations.
\end{abstract}

Keywords Cross-modal associations · Haptic perception · Color

Cross-modal correspondences can be generally defined in terms of nonarbitrary associations across perceptual modalities. Although these types of associations have been increasingly studied in the past years, their specific nature is still

N. Slobodenyuk $(\bowtie) \cdot$ L. Ghanem

Department of Psychology, American University of Beirut, Riad

El-Solh, P.O. Box 11-0236, Beirut 1107 2020, Lebanon

e-mail: ns74@aub.edu.lb

Y. Jraissati

Department of Philosophy, American University of Beirut,

Beirut, Lebanon

A. Kanso - I. Elhajj

Department of Electrical and Computer Engineering, American

University of Beirut, Beirut, Lebanon unclear (see Spence, 2011, and Deroy \& Spence, 2013a, for review). A large body of research shows that in addition to the relatively infrequent synesthetic cross-modal associations, mappings between sensations from different modalities are common in general population and particularly strong in children. Several accounts of cross-modal associations found in general population have been proposed in the literature. The neonatal synesthesia hypothesis traces adult cross-modal associations to infancy and presumes a shared origin of synesthetic, infant, and adult cross-modal experiences. The hypothesis is based on the evidence that functional connections between and within sensory areas found at birth, to some extent persist into adulthood, and proposes that these connections account for the cross-modal associations. Synesthetic associations, according to the hypothesis, could be explained by selective exaggeration of these connections (Spector \& Maurer, 2009). Such unifying account of the synesthetic perception, childhood, and adult cross-modal associations that assumes shared origin and continuity of the common crossmodal associations and rare cases of synesthesia, however, has been placed under scrutiny due to either partial empirical evidence or its radical interpretation. Weak points of the hypothesis that have been pointed out pertain to the inference of cross-modal experience in infants on the basis of increased connectivity, as well as substantial qualitative differences between adult cross-modal mappings and synesthetic associations (see Deroy \& Spence, 2013b, for the extended argument).

Other accounts of cross-modal correspondences do not necessarily reject the whole idea of the early low-level mechanisms but presume that "although cross-modal correspondence may arise from sensory mechanisms in infants, these correspondences reflect postsensory (meaning-based) mechanisms in adults" (Martino \& Marks, 2001, p. 64). The idea of the semantic nature of cross-modal associations is grounded in the work of Osgood and his colleagues (Osgood, 1952; 
Osgood \& Suci, 1955; Osgood, Suci, \& Tannenbaum, 1957) who proposed that perceptual experiences are mapped into multidimensional semantic space with the main empirically singled dimensions of potency, activity, and evaluative meaning. Each dimension is represented by a range of scales defined by polar adjectives. For example, large-small, heavylight, thick-thin, and strong-week represent the potency dimension. Fast-slow, active-passive, hot-cold, sharp-dull, and angular-rounded are examples of the activity dimension. Good-bad, sweet-sour, sweet-bitter, fragrant-foul, and beautiful-ugly represent the evaluative dimension. Factor analyses conducted by Osgood \& Suci (1955) have also shown that some scales are related to more than one dimension. For example, hard-soft and rough-smooth mainly represent potency, but they also are related to evaluative meaning. Similarly, red-green and tense-relaxed fall within the activity dimension but represent evaluative dimension as well. The idea of the semantic nature of cross-modal associations has taken several forms in the literature. Martino \& Marks (1999b) put forward a semantic coding hypothesis ( $\mathrm{SCH}$ ) according to which crossmodal correspondences result from perceptual experiences being recorded into a single abstract representation in a sematic network that captures correspondence between the two stimuli features. Woods, Spence, Butcher, \& Deroy (2013) use the notion of semantic hypothesis that is more closely related to the work of Osgood and refers to the assumption that cross-modal associations occur due to the shared conceptual dimension between stimuli from different modalities (e.g., high brightness is mapped to high pitch due the common dimension of activity). Woods et al. note some criticisms of such semantic hypothesis. First, the plurality of dimensions makes the explanatory power of the semantic hypothesis unlimited, because it might be possible to find a common dimension of any two objects and explain their matching on the basis of this dimension. Second, it is possible that the semantically determined associations emerge as a byproduct of the forced choice method and the participants' effort to identify the conceptual association that the experimenter had in mind. Finally, Woods et al. also question whether dimensions proposed by Osgood \& Suci (1955) should be the only ones used to explain cross-modal associations or whether the idea of a more abstract semantic network proposed by Martino \& Marks (1999b) could provide a better framework for explaining the semantic nature of cross-modal associations.

Some empirical findings challenge the semantic explanation for at least some cross-modal correspondences and point to the possibility of associations that arise from exposure to the immediate sensory environment. For example, Parise, Knorre, \& Ernst (2014) have recently proven that the mapping between high-pitched sounds and higher locations in space could be related to the spatial locations of the sound sources in the natural environment. By analyzing recordings from wearable directional microphones attached to the heads of the participants who normally moved in various environments, Parise et al. were able to conclude that the sources of the high-pitched sounds, in fact, tend to be elevated in the natural environment and, therefore, mapping between pitch and spatial height is likely to be grounded in this statistical regularity. The statistical regularities hypothesis and multisensory statistical learning, in particular, have received a lot of attention in the literature (e.g., Glicksohn \& Cohen, 2013; Mitchel \& Weiss, 2011); however, in many cases, the statistical origin of cross-modal associations is difficult to prove due to the need for substantial environment sampling. The hypothesis, at least theoretically, was extended to explain more complex second-order correspondences. For example, mapping between the high pitch and angularity of objects could be due to the fact that harder objects resonate at higher frequencies and tend to scatter into sharper pieces than softer objects when broken (Parise \& Spence, 2012). Similarly, mapping between high lightness and high pitch may be considered a result of coupling between the light-from-above heuristic and the higher probability of the smaller high-pitched objects to be found near the sources of ambient light (Spence \& Deroy, 2012). Spence and Deroy also suggest that due to the transitive nature of cross-modal correspondences, it is possible that the correspondence between lightness and pitch is acquired once lightness/size and size/pitch regularities are learned.

The idea of different kinds of cross-modal correspondences offered by Spence (2011) to some extent reconciles the accounts and removes the need for one overarching explanation for all cross-modal associations. According to Spence's classification, cross-modal associations can be 1) statistical, 2) structural, and 3) semantically mediated. Statistical crossmodal correspondences are the ones that reflect correlations between the stimuli dimensions in the natural environment (e.g., pitch/ size correspondence on the basis of object's resonant frequency). Structural correspondences are the ones that are grounded in brain connectivity in infancy and reflect organizational peculiarities of perceptual systems. The best example here seems to be the shared cortical representation of the magnitude regardless of the stimulus dimension (see Walsh, 2003). Finally, semantically mediated cross-modal correspondences might arise due to linguistic overlap between notions used to describe different stimuli dimensions. Spence gives the use of "high" and "low" to describe spatial height and tone's pitch as an example of such semantically mediated correspondences. Perhaps, such understanding of semantically mediated origin of cross-modal associations does not fully mirror the semantic hypothesis discussed earlier but rather adds a lexical aspect to it. In light of Parise et al.'s (2014) results, mapping between spatial height and pitch presents a particularly interesting case, because it can be viewed as a semantically mediated association but also as a statistical one. However, one might also argue that we pick up environmental regularities earlier than we acquire language; therefore, 
our immediate sensory environment is the source of this association.

The present study focuses on the cross-modal correspondences between color and tactile sensations in general population. Previously, color has been found to be associated in a systematic manner with smells (Gilbert, Martin, \& Kemp, 1996; Kim, 2013; Schifferstein \& Tanudjaja, 2004), tastes (Spence, Levitan, Shankar, \& Zampini, 2010), sounds (pitch-lightness mappings in Ward, Huckstep, \& Tsakanikos, 2006; pitch-lightness and ascending/descending melodic intervals to lightness mappings in Hubbard, 1996), and more complex auditory stimuli, such as composed music pieces (Palmer, Schloss, Xu, \& Prado-León, 2013). In some cases, the associations with color can be explained through emotional mediation (Palmer, Schloss, Xu, \& Prado-León, 2013), and linguistic (Spector \& Maurer, 2011); in other cases, a direct unmediated connection can be assumed. Whereas literature on the associations between color and stimuli from the mentioned sensory modalities is abundant, only few studies have pointed to the possibility of cross-modal associations between color and tactile sensations. Martino \& Marks (1999a) showed systematic matching between visual lightness (black, white) and vibrotactile frequency (low, high). Most recently, Ludwig \& Simner (2013) have also revealed nonrandom associations between color and tactile stimuli. They asked participants to select a color that matches the tactile sensations of roughness/ smoothness, hardness/softness, and pointiness/roundness. Each of the three sensation groups contained six stimuli on a sensation continuum. The results showed systematic associations between smoothness, softness, roundness, and luminance, as well as associations of smoothness and softness with chroma. The authors also indicated that some tactile sensations were associated with specific colors (e.g., roughness was associated with color brown).

The present research intended to replicate the findings of the Ludwig \& Simner's (2013) study while expanding the range of tactile sensations and using haptically rendered virtual stimuli rather than real objects and surfaces. Our research includes not only rough/smooth and hard/soft properties studied by Ludwig and Simner, but also three additional categories of substance properties that can be experienced in a tactile manner and rendered through haptic interfaces. These are heavy/light, elastic/inelastic, and adhesive/nonadhesive. Note that Ludwig and Simner had a third tactile category_ - pointed/ round; however, this category was not included in our study for the purpose of limiting the haptic experiences to substance properties. Another reason for eliminating this characteristic is that Ludwig and Simner viewed pointiness/roundness as one property with pointy on one side of the continuum and round on the other side; it is, however, unclear whether we actually perceive pointy/round as a property rather than independent shape characteristic, and whether we actually perceive a sphere, for example, to be on the other side to a triangle on the continuum. This is a particularly problematic issue considering that visual shapes are systematically associated with information from other modalities (e.g., Albertazzi \& Da Pos, 2012; Spector \& Maurer, 2011). Taking into account the evidence that tactile and visual shape processing converge in the lateral occipital complex (Amedi et al., 2002), one might also consider the possibility of the spillover of visual shape and color associations to tactile shape and color association. Given this assumption, it is possible that the color and pointy/round associations studied by Ludwig and Simner actually reveal the mappings between colors and specific shapes, not the characteristic of pointiness/roundness that can change gradually. Therefore, pointy/round was not included in our study. Due to the peculiarities of haptic interfaces, inclusion of the shape also would have required contextualization by adding visual information, and any visual input associated with the haptic feedback was purposefully eliminated to limit additional sensory interference.

There are some benefits of using virtual haptic stimuli compared with real stimuli. Haptic rendering of substance properties is relatively well researched and literature offers a large variety of algorithms for rendering roughness, weight, viscosity, stiffness, and softness (see Bicchi, Buss, Ernst, \& Peer, 2008, for review). Phenomenally, sensations of substance properties of virtual objects are comparable to sensations resulting from interaction with real objects (see Culbertson, Unwin, \& Kuchenbecker, 2014; Unger, Hollis, \& Klatzky, 2011, for real vs. virtual roughness comparison); however, some substance properties have been researched more than others, and the comparison between real and virtual object experiences often is drawn only on the basis of measurements of quantitative abilities of the user. Similarly to tactile experiences with real objects, haptic experiences of virtual objects can be enhanced with relevant visual (Drewing, Ernst, Lederman, \& Klatzky, 2004; Jones, Bokinsky, Tretter, \& Negishi, 2005) and auditory cues (Kitamura et al., 2006), and haptic recognition can be improved with multisensory input. The benefit of using virtual haptic stimuli is mainly in the possibility of representing only one property of the object and eliminating the interference of other properties. In Ludwig \& Simner's (2013) study, which is most relevant to our research, sandpaper was used for rough/smooth stimuli, foam material for hard/soft, and wooden shapes for pointy/round. It is possible that additional factors (e.g., temperature of the material) and associations with the material itself influenced the matching between the target sensation and color. We also view the use of the tactile stimuli rendered through haptic interface as of particular importance to the field of development of haptic color rendering systems for the visually impaired. While the field of sensory substitution systems is developing rapidly, the existing haptic color rendering systems have not made use of the cross-modal naturally biased associations. 


\section{Method}

Participants

Fifty-four students participated in the study as part of their introduction to psychology course credit at the American University of Beirut. All participants had normal or corrected-to-normal vision and normal color vision. Color vision of the participants was confirmed on the basis of the response to Ishihara plates prior to the experiment. The study was approved by the Institutional Review Board (IRB) at the American University of Beirut.

\section{Stimuli and Design}

SensAble PHANTOM OMNI ${ }^{\circledR}$ haptic device was used to render haptic stimuli. The device with the assigned axes is presented in Fig. 1. The maximum exertable force by Phantom Omni is equal to $3.3 \mathrm{~N}$; therefore, the forces used for each stimulus were limited to the $[-3.3 \mathrm{~N} ; 3.3 \mathrm{~N}]$ range. Five haptic sensations were rendered: 1) roughness, 2) hardness, 3) heaviness, 4) elasticity, and 5) adhesiveness. Six levels of intensity of each sensation were created and adjusted to correspond to perceptual equidistance.

The rendering of roughness The roughness sensation was created by applying a sine-wave or saw-tooth shape forces (Equations 1 and 2), which are related to the joystick's planar motion on a virtual surface.

$F_{y}=\left|\mathrm{K}_{\mathrm{a}} \times \sin \left(2 \pi K_{f} \sqrt{x^{2}+z^{2}}\right)\right| \quad$ or

$F_{y}=\left|\mathrm{K}_{\mathrm{a}} \times \operatorname{sawtooth}\left(2 \pi K_{f} \sqrt{x^{2}+z^{2}}\right)\right|$

By altering the frequency $\left(K_{f}\right)$ of these waves and their amplitude $\left(K_{a}\right)$, we created different sensation levels that

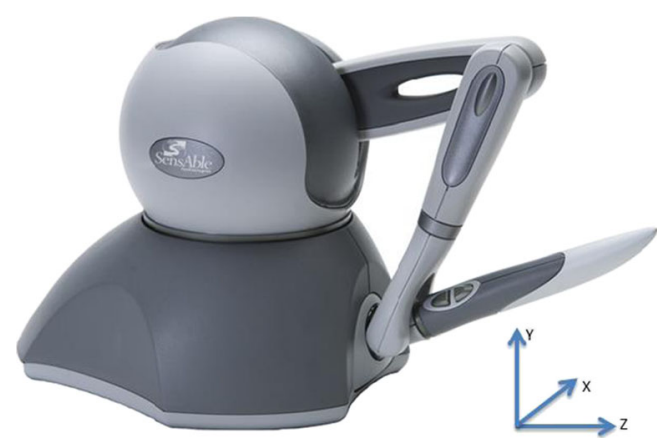

Fig. 1 SensAble PHANToM OMNI ${ }^{\circledR}$ haptic device simulate the surfaces with different degrees of roughness. The forces applied for each level of roughness sensation are summarized in Table 1.

The virtual surface was located at height $\mathrm{Y}=0$. Below this elevation, a counter reaction force was applied at the joystick's tip proportional to the level of surface penetration

$$
F_{y_{\text {counter }}}=-\frac{Y}{k_{c}}
$$

where $\mathrm{Y}$ is the joystick's height and $k_{c}$ is a constant (equal to 20) used to dampen the counter force amplitude. Therefore, the total force felt by the participant was equal to

$$
F_{y_{\text {total }}}=F_{y}+F_{y_{\text {counter }}}
$$

and this force was applied in the vertical direction only (along y axis).

The rendering of hardness The hardness sensation was rendered by applying a force related to the joystick's vertical movement (horizontal planar motion had no effect) on an imaginary plane in space. The vertical force applied was equal to:

$F_{y}=-\frac{Y}{k}$

where $k$ is a constant used to change the magnitude of the exerted force. The parameters used to create 6 levels of hardness sensations are following: Level 1 (softest): $k=20$; level 2: $k=14$; level $3: k=8$; level $4: k=5$; level $5: k=3$; level 6 (hardest): $k=0.7$.

With a certain exerted pressure, a soft surface could be easily penetrated with a minimum repelling force, whereas the hard surface was more solid and harder to penetrate because of the maximum repelling force applied. Such peculiarities were considered in the instruction to the task and

Table 1 Roughness sensation forces for six levels of intensity rendered on the basis of perceptual equidistance

\begin{tabular}{llll}
\hline Level & Function used & $\mathrm{K}_{\mathrm{a}}$ & $\mathrm{K}_{\mathrm{f}}$ \\
\hline 1 (Smooth) & sine & 0.8 & 0.02 \\
2 & sine & 0.9 & 0.03 \\
3 & sine & 0.8 & 0.05 \\
4 & sine & 1 & 0.08 \\
5 & sawtooth & 1.3 & 0.5 \\
6 (Rough) & sine & 1.5 & 0.32 \\
\hline
\end{tabular}


controlled during the practice stage as well as the experimental stage.

The rendering of heaviness The heaviness sensation was produced by applying a constant downward force $F_{y}=-k$. The force applied in heaviest level was stronger than that applied in the lighter ones. This feeling simulated lifting a body of a certain weight off the ground. By releasing a button on the stylus of the haptic device, the user could remove the force applied thus creating the illusion of dropping the lifted body. The parameters used to create 6 levels of heaviness sensations are following: Level 1 (lightest): $k=0.8$; level 2: $k=1.1$; level 3: $k=1.5$; level 4: $k=2$; level 5: $k=2.7$; level 6 (heaviest): $k=3.3$.

The rendering of elasticity The elasticity sensation was created by applying a force that increased in intensity as the user clicked and pulled the joystick in $3 \mathrm{~d}$ space. The direction of forces applied opposed the user's motion. Moreover, the forces applied in case of most elastic stimulus had lower magnitudes than those applied in less elastic ones. The force applied was equal to

$F=\frac{\Delta_{p o s}}{k}$

where $k$ is a stimulus level constant used to change the magnitude of the applied force, and $\Delta_{\text {pos }}$ (Equation 7) is the displacement vector between the joysticks position when the participants clicked the button and the current one.

$\Delta_{\text {pos }}=\left[\begin{array}{lll}X & Y & Z\end{array}\right]_{\text {button click }}-\left[\begin{array}{lll}X & Y & Z\end{array}\right]_{\text {current }}$

The parameters used to create 6 levels of elasticity sensation are following: Level 1 (most elastic): $k=50$; level 2: $k=$ 25; level 3: $k=14.3$; level 4: $k=6.5$; level $5: k=2.5$; level 6 (most inelastic): $k=0.8$.

The rendering of adhesiveness The adhesiveness sensation was created by making a virtual horizontal plane sticky. While moving away from the surface, a downward force ( $k$ is a stimulus level constant) was applied on the participant's hand and increased in intensity with the increase of the distance from the virtual plane (Equation 8).

$F_{y}=-\frac{Y}{k}$

If the user moved further away from the surface, the applied force was eventually cancelled and he/she could pop free. The intensity level of adhesiveness could be changed by increasing the force magnitude or by changing the vertical Y position at which the force was released (breakpoint). The parameters used to create 6 levels of adhesiveness and the related breakpoints are following: Level 1 (least adhesive): $k$ $=8$, breakpoint $=10$; level $2: k=6$, breakpoint $=10$; level $3: k$ $=5$, breakpoint $=14$; level $4: k=2$, breakpoint $=14$; level $5: k$ $=1 / 2$, breakpoint $=30$; level 6 (most adhesive): $k=1 / 3$, breakpoint $=44$.

The virtual surface was created by applying a force opposing the joystick's downward motion, where

$$
F_{y_{\text {counter }}}=-\frac{Y}{k_{c}}, k_{c}=4 ;
$$

hence, the force felt by the participant was equal to

$$
F_{y}=\left\{\begin{array}{cl}
-\frac{Y}{k}, & Y \geq 0 \\
-\frac{Y}{k_{c}}, & Y<0
\end{array}\right.
$$

Each level of intensity for every sensation type was presented in the final experiment 3 times. This resulted in 18 stimuli per sensation type and an overall of 90 stimuli. Five sensation types were presented to the participants in random order; the presentation of different levels within sensation type also was randomized.

\section{Procedure}

Participants were seated in front of a 17" computer screen (Fujitsu Siemens Computers GmbH, LCD Color Monitor, ScenicView B17-5, resolution $1280 \times 1024$ pixels) at a distance of $50 \mathrm{~cm}$. The screen presented an HSV color wheel and a brightness slider on the right side of the wheel (Fig. 2). A color preview area was added to the side of the slider to allow for the preview and the possibility of modification of the choice before it was submitted. The HSV color wheel, as opposed to other color spaces and color space representations, was chosen due to the visual familiarity to the participants. The haptic device was placed on the right side if the participant was right-handed and the mouse on the left side. If the participant reported left-handedness, the sides for the haptic device and the computer mouse were changed.

Participants were given the task to select a color on the color wheel that matches the experienced haptic sensation. They also were instructed to concentrate on the feeling itself rather than think about the resemblance to actual objects. When participants took a lot of time to choose the color after experiencing a target haptic stimulus, they were once more encouraged by the experimenter not to overthink and to focus on sensation matching. 


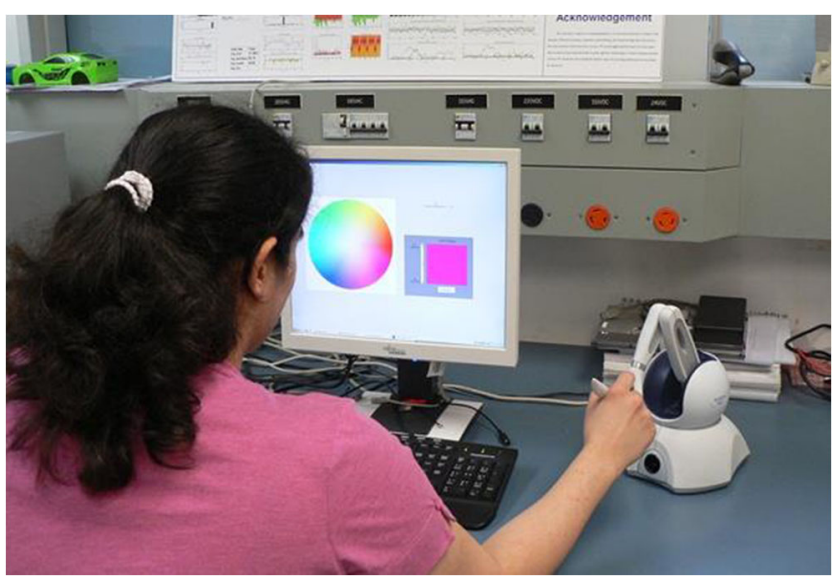

Fig. 2 Experimental setup

The setup allowed the participants to experience the haptic sensation with their dominant hand and make the color choice using the computer mouse with their nondominant hand. The color wheel and the brightness slider were large enough for the participant to immediately adapt to using the mouse with the nondominant hand.

Instructions on how to experience a particular sensation type were given at the beginning of each sensation block. Instructions to roughness, hardness, elasticity, and adhesiveness blocks involved interaction with virtual surfaces and exploratory procedures corresponding to those that are used to identify roughness, hardness, elasticity, and adhesiveness of real objects. Instructions to heaviness sensation involved lifting and dropping a virtual object. Each sensation block started with the instruction, followed by two trials depicting the extremes of the sensation in particular sensation block. For example, on the hardness block, participants were able to sense the hardest stimulus they would experience during the experimental stage and the softest one. They were explicitly informed about the fact that they are sensing the extremes, and that the experiment will involve the extremes and the intermediate sensations. During the experimental stage that followed the instructions and the familiarization trials for each sensation, participants on average spent 11 seconds exploring each stimulus. The experimenter stayed beside the participant the whole duration of the experiment to give instructions on how to experience a sensation before sensation blocks, to make sure that the instructions are being followed, and to attend to the needs of position adjustment for fatigue prevention.

\section{Results}

Treatment of the Data

Individual responses for all levels of sensations for five haptic stimuli were projected to CIEL*a*b* color space which is uniform and can be considered a "simple example of a color appearance model" (Fairchild, 2013, p. 201). The CIEL*a*b* coordinates were obtained using a two-step conversion method: RGB to XYZ and XYZ to L*a*b* (Lindbloom, 2014). The resulting space could be considered an approximation of CIEL $* a * b *$ color space as the monitor characteristics were not taken into account. All further analyses were conducted using these CIEL* $\mathrm{a}^{*} \mathrm{~b} *$ coordinates.

Each level of intensity of the five sensation types was presented to the participant three times. The participant responses for the three identical stimuli were not averaged, because they represent data points in the three-dimensional color space and could be entirely different in hue but share the properties of brightness or saturation. To avoid data loss, these responses were treated as an independent input.

\section{Mapping Haptic Stimuli in Color Space}

In attempt to identify the differences between the mapping of five haptic stimuli of different intensities (levels) in color space on the basis of participant color choices, K-means and Kmedoids clustering algorithms were used. They proved inefficient and did not show clear level-dependent clusters; therefore, a method of comparing distance to centroids of data points (responses) in stimuli of different levels was used. Centroids for levels 1 and 6 representing extremes of the sensations, as well centroids for intermediate levels 3 and 4 were calculated. $T$ tests and Mann-Whitney tests were conducted to test whether distances to centroid 1 (i.e., centroid of level 1 responses) and centroid 6 , respectively, were different for levels 1 and 6 responses for each of the five stimuli (roughness, hardness, heaviness, elasticity, and adhesiveness). Subsequently, we tested whether distances to centroid 3 and centroid 4, respectively, were different for levels 3 and 4 responses for the five haptic stimuli.

The results of comparison of distance to centroids for level 1 and 6 sensations (Table 2) and level 3 and 4 sensations (Table 3 ) show that level 1 responses were significantly closer to centroid 1 than level 6 responses and level 6 responses were significantly closer to centroid 6 than level 1 responses, whereas only in a few cases level 3 responses were closer to centroid 3 than level 4 responses, and none of the level 4 responses were significantly closer to centroid 4 than level 3 responses. Such pattern indicates that the color choices were related to the intensity of the haptic stimuli because the responses for the maximally different levels of sensations ( 1 and 6 ) were situated in more distinctive areas of the color space and as intensity of sensation became more similar, this difference decreased.

Haptic Stimuli and Brightness

The highest average brightness per stimulus level was 84.06 in CIEL*a*b*. Typical sky blue in Munsell is around 5PB 7/10 
Table 2 Distances to Centroids $1 \& 6$

\begin{tabular}{|c|c|c|c|c|}
\hline & Distance to Centroid 1 & & Distance to Centroid 6 & \\
\hline Stimulus & Test of difference & Effect size & Test of difference & Effect size \\
\hline Heavy/light & $U=4267, Z=-10.54^{*}$ & $r=0.59$ & $\mathrm{t}(315.18)=5.67^{* *}$ & $r=0.30$ \\
\hline Rough/smooth & $U=6785.5, Z=-7.52^{*}$ & $r=0.42$ & $U=11266.5, Z=-2.20^{*}$ & $r=0.12$ \\
\hline Hard/soft & $U=4914, Z=-9.74^{*}$ & $r=0.54$ & No difference & \\
\hline Elastic/inelastic & $\mathrm{t}(322)=-10.15^{* *}$ & $r=0.49$ & $U=10848, Z=-2.70^{*}$ & $r=0.15$ \\
\hline Adhesive/nonadhesive & $U=3955, Z=-10.87^{*}$ & $r=0.60$ & $U=10583, Z=-3.01^{*}$ & $r=0.17$ \\
\hline
\end{tabular}

* Mann-Whitney test used to test for differences

** Independent samples $t$ test used to test for differences

(hue 5PB, value 7, chroma 10), which is approximately 71.6 brightness value in CIEL*a*b*; 84.06 is, therefore, a bit over one Munsell step further in brightness. The lowest average brightness per stimulus level was 33.01 in CIEL*a*b*. This approximately amounts to value 3 in Munsell (30.77 in CIEL*a*b*), which is a brightness level of a typical brown.

The assumption of homogeneity of variance was not met; therefore, the relationship between the intensity of haptic stimuli and brightness was examined using Welch's F. An adjusted formula for $\omega^{2}$ was used to test for effect sizes.

$\omega^{2}=\frac{\mathrm{df}_{\text {bet }}\left(\mathrm{F}_{\mathrm{w}}-1\right)}{\mathrm{df}_{\text {bet }}\left(\mathrm{F}_{\mathrm{w}}-1\right)+\mathrm{N}_{\mathrm{T}}}$

The results of the conducted analysis (Table 4) indicate that there is an overall difference between levels of haptic stimuli in terms of brightness and that the color choices for the least intense haptic stimuli were the brightest, whereas the color choices for the most intense haptic stimuli were the least bright (Fig. 3).

\section{Haptic Stimuli and Chroma}

On the basis of Fairchild's (2013) recommendation, chroma was calculated from CIEL*a*b* color space coordinates using

$C=\sqrt{a^{2}+b^{2}}$

The lowest average chroma per stimulus level was 40.39 in CIEL*a*b*. A typical sky blue in Munsell (5PB 7/10) approximately amounts to chroma 40.6 in CIEL*a*b*. A pale bright yellow is $5 \mathrm{Y} 9 / 4$ or $9 / 6$, which amounts in CIEL*a*b* to approximate chroma of 31.45 and 45.54 , respectively. The highest average chroma per stimulus level was 66.9. A typical red in Munsell is around 5R 4/14, which amounts in CIEL*a*b* to approximately 66.7 chroma value.

Considering that the assumption of homogeneity of variance was not met, the relationship between the intensity of haptic stimuli and chroma was examined using Welch's F. An adjusted formula for $\omega^{2}$ was used to test for effect sizes. $\omega^{2}$ represents the total variance in the dependent variable (chroma) that is accounted for by the independent variable (level). In addition, post-hoc tests were conducted with Games-Howell corrections.

The results of the conducted analysis (Table 5) indicate that there is an overall significant difference between levels of haptic stimuli in terms of chroma and that the color choices for the least intense haptic stimuli were associated with the lowest degree of chroma. The highest degree of chroma, on the other hand, was characteristic to intermediate levels of haptic stimuli intensity (Fig. 4).

Table 3 Distances to Centroids $3 \& 4$

\begin{tabular}{|c|c|c|c|c|}
\hline & Distance to Centroid 3 & & Distance to Centroid 4 & \\
\hline Stimulus & Test of difference & Effect size & Test of difference & Effect size \\
\hline Heavy/light & $\mathrm{t}(322)=-2.59^{* *}$ & $r=0.14$ & No difference & \\
\hline Rough/smooth & $U=10725, Z=-2.84^{*}$ & $r=-0.22$ & No difference & \\
\hline Hard/soft & No difference & & No difference & \\
\hline Elastic/inelastic & No difference & & No difference & \\
\hline Adhesive/nonadhesive & $\mathrm{t}(322)=-3.07^{* *}$ & $r=0.17$ & $\mathrm{t}(322)=-2.42^{* *}$ & $r=0.13$ \\
\hline
\end{tabular}

* Mann-Whitney test used to test for differences

** Independent samples $t$ test used to test for differences 
Table 4 Haptic stimuli and brightness

\begin{tabular}{lll}
\hline Stimulus & Welch's F test & Effect size \\
\hline Heavy/light & $\mathrm{F}_{\mathrm{W}}(5,147.26)=77.19^{*}$ & $\omega^{2}=0.54$ \\
Rough/smooth & $\mathrm{F}_{\mathrm{W}}(5,147.75)=30.14^{*}$ & $\omega^{2}=0.31$ \\
Hard/soft & $\mathrm{F}_{\mathrm{W}}(5,147.58)=42.20^{*}$ & $\omega^{2}=0.38$ \\
Elastic/inelastic & $\mathrm{F}_{\mathrm{W}}(5,144.99)=52.39^{*}$ & $\omega^{2}=0.44$ \\
Adhesive/nonadhesive & $\mathrm{F}_{\mathrm{W}}(5,145.37)=55.61^{*}$ & $\omega^{2}=0.45$
\end{tabular}

* Significant at the $p<0.01$ level

Haptic Stimuli and Hue

According to Fairchild (2013, p.204), in CIEL*a*b*, and under daylight illumination, unique red lies approximately at
Table 5 Haptic stimuli and chroma

\begin{tabular}{lll}
\hline Stimulus & Welch's F test & Effect size \\
\hline Heavy/light & $\mathrm{F}_{\mathrm{W}}(5,449.48)=8.80^{*}$ & $\omega^{2}=0.04$ \\
Rough/smooth & $\mathrm{F}_{\mathrm{W}}(5,450.20)=4.90^{*}$ & $\omega^{2}=0.01$ \\
Hard/soft & $\mathrm{F}_{\mathrm{W}}(5,449.69)=13.27^{*}$ & $\omega^{2}=0.06$ \\
Elastic/inelastic & $\mathrm{F}_{\mathrm{W}}(5,449.88)=10.41^{*}$ & $\omega^{2}=0.05$ \\
Adhesive/nonadhesive & $\mathrm{F}_{\mathrm{W}}(5,449.78)=25.62^{*}$ & $\omega^{2}=0.10$
\end{tabular}

${ }^{*}$ Significant at the $p<0.01$ level

$24^{\circ}$, unique yellow at $90^{\circ}$, unique green at $162^{\circ}$, and unique blue at $246^{\circ}$. We have opted for a more specific pigment system by Bruce MacEvoy (2009) that provides hue coordinates of 24 criterion pigments in CIEL*a*b*.
Fig. 3 Brightness of color choices for different levels of intensity of haptic stimuli
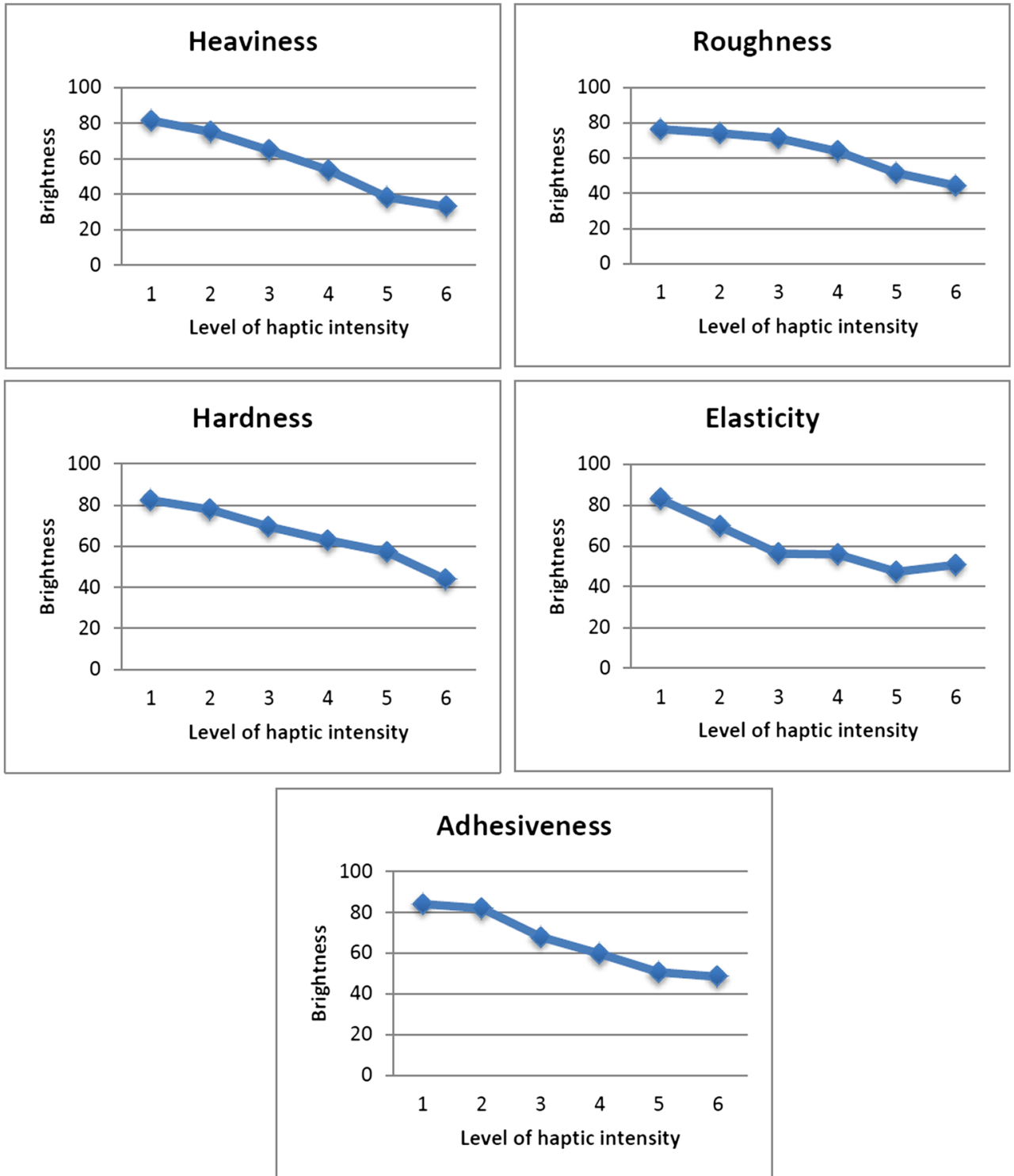
Fig. 4 Chroma of color choices for different levels of intensity of haptic stimuli
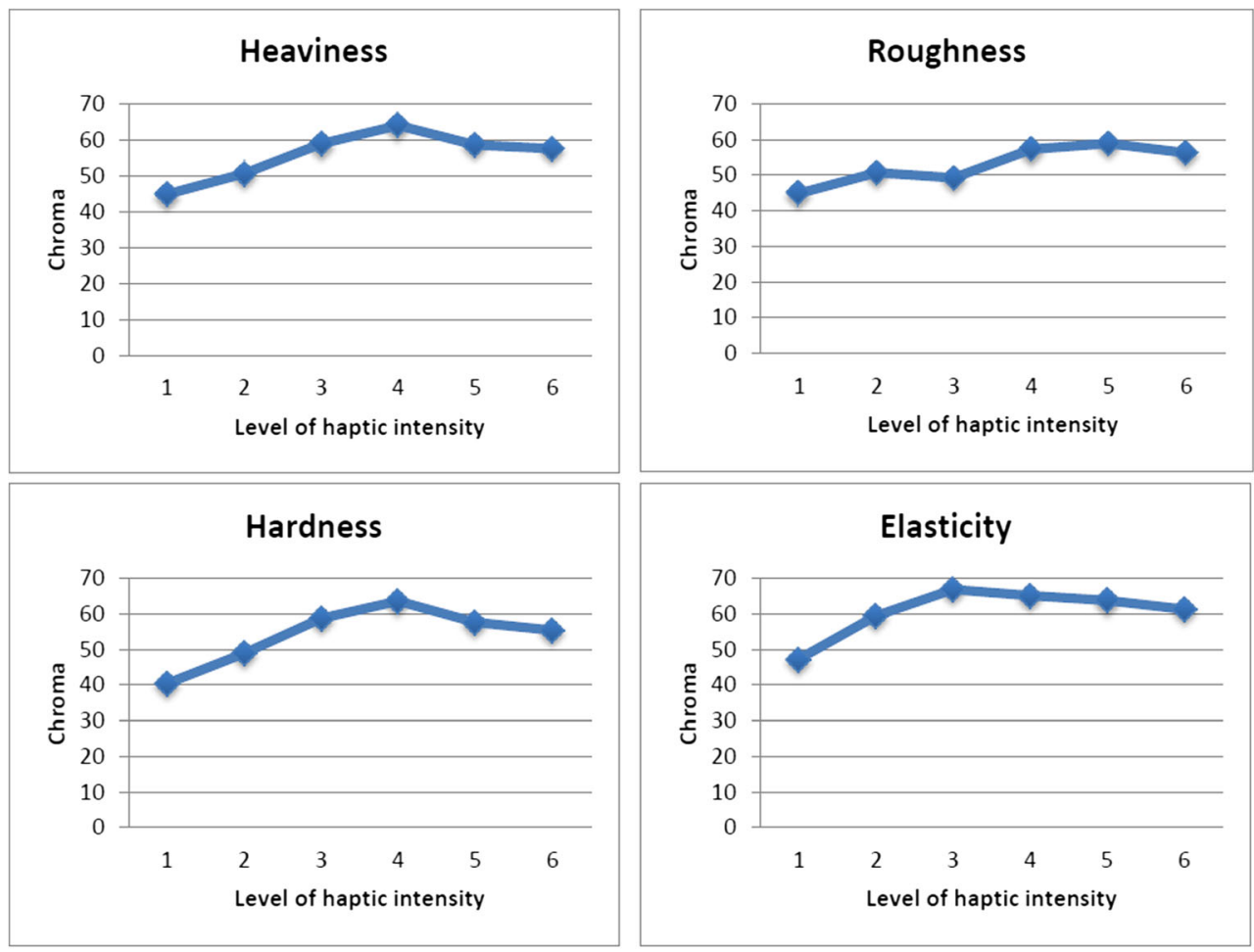

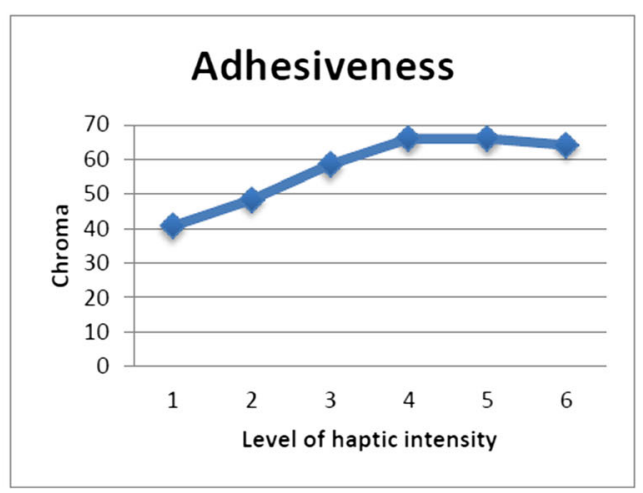

The histograms of the CIEL*a*b* data points (i.e., participant responses) for level 1 and level 6 with an interval of 15 degrees were projected onto the $\mathrm{a}^{*} \mathrm{~b}^{*}$ plane with 24 pigment indications (Figs. 5, 6, and 7). The comparison of the distribution of the data points revealed high similarity between various haptic stimuli for level 1 , such similarity was even more salient in case of level 6 responses. Manhattan distance normalized by the number of intervals was used to calculate such similarity. On average the difference between level 1 smoothness, softness, lightness, elasticity, and adhesiveness was in the range between 2.25 and 2.91 data points with an exception of the elasticity difference with smoothness and lightness in 3.75 data points. On average, the difference between level 6 smoothness, softness, lightness, and elasticity was in the range between 2.08 and 2.5 with an exception of elasticity difference with lightness was 3.5 data points. Adhesiveness was different from all other stimuli in a range of 2.83 to 3.5 data points.
The comparison between the distribution of the data points for level 1 and level 6 stimuli also revealed that level 1 responses were distributed more evenly across the hue plane in all haptic sensations with smaller hue biases than level 6 stimuli. Table 6 depicts the comparison between the maximum number of data points per interval, minimum number of data points per interval, and a standard deviation for the five haptic stimuli. Due to more even distribution of level 1 responses in hue plane, biases towards specific hue spectra were smaller than in case of level 6 responses across all haptic sensations. Considering that the largest number of data points per interval in level 1 and 6 across all haptic stimuli was 35, for the purpose of the present analysis, the size of the hue biases was defined the following way: large bias [30, 35] data points, medium bias $[20,30)$ data points, and small bias $[15,20)$ data points. Accordingly, hue biases for level 1 and 6 responses are depicted in Table 7 and visualized in Figs. 5, 6, and 7. 


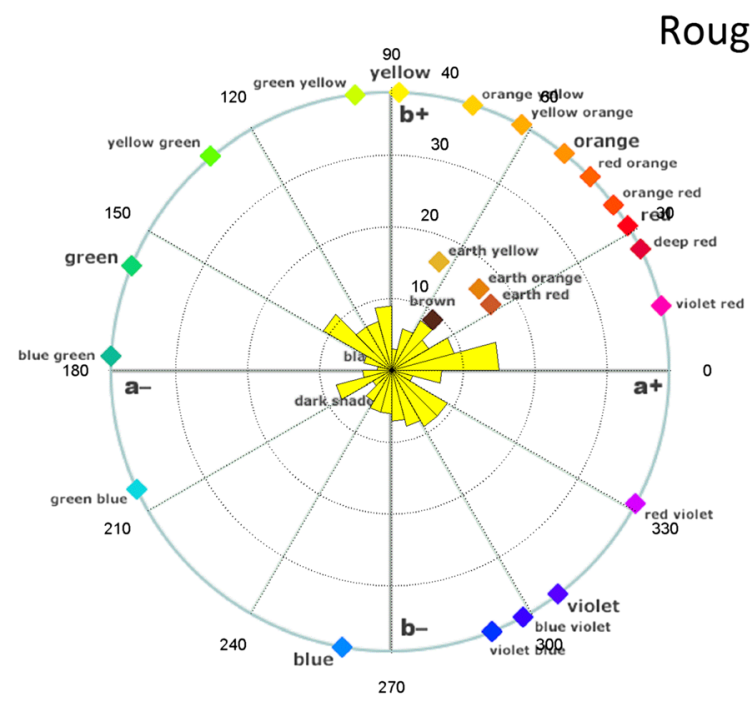

level 1

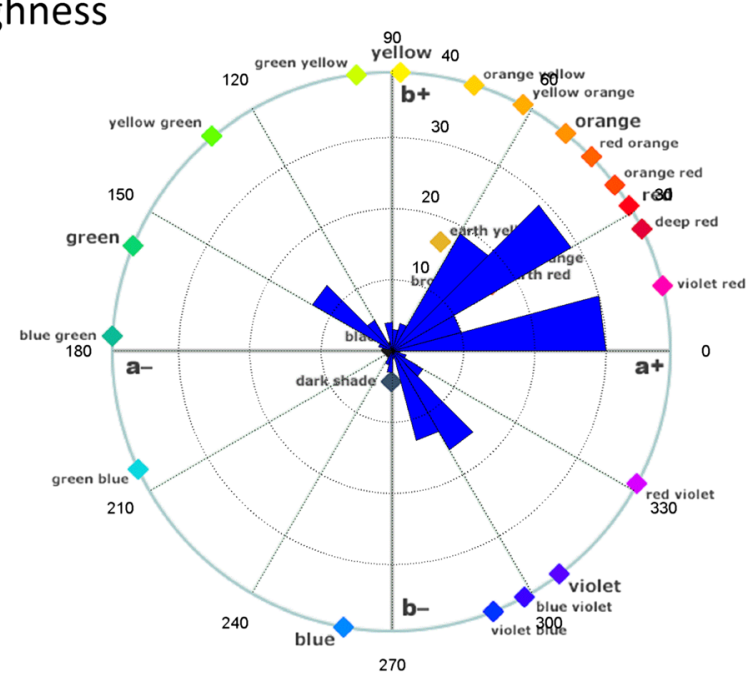

level 6

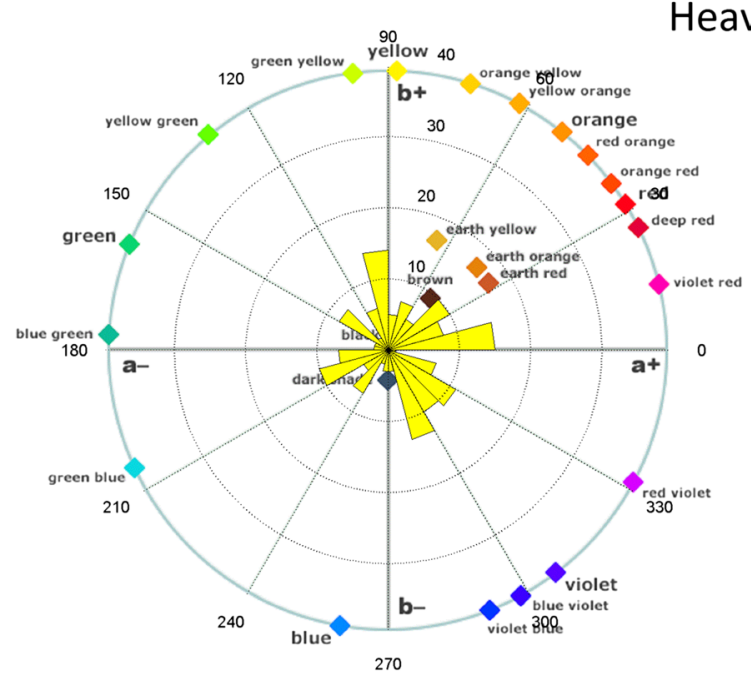

level 1

Heaviness

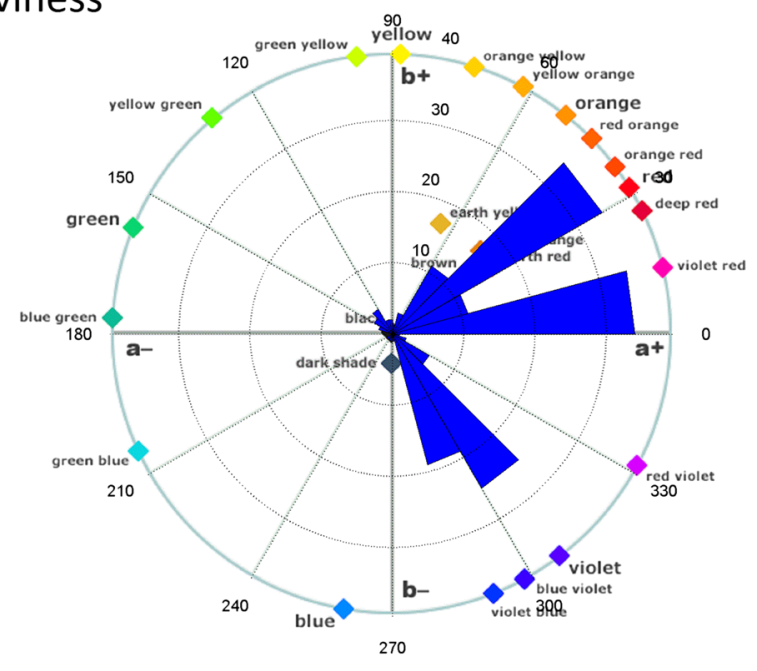

level 6

Fig. 5 Histograms of the CIEL*a*b* data points (i.e., participant responses) for level 1 and level 6 of the roughness and heaviness stimuli with an interval of 15 degrees projected onto the $a * b *$ plane. CIEL*a*b* hue plane with pigment indications courtesy of Bruce MacEvoy

\section{Discussion}

The present research showed systematic cross-modal matching between the properties of haptic stimuli and color dimensions in a general population. Similarly to other studies on cross-modal associations with color where brightness emerged as a salient characteristic (e.g., Albertazzi \& Da Pos, 2012; Kim, 2013), we have found a strong relationship between brightness and intensity of haptic stimuli across all haptic sensations. The most intense haptic sensations were associated with the color choices of the lowest brightness. The heaviest, the roughest, the hardest, the most inelastic, and the most adhesive stimuli were the least bright. Brightness increased as heavy stimuli became lighter, rough stimuli became smoother, hard stimuli became softer, inelastic stimuli became more elastic, and adhesive stimuli became less adhesive. This is consistent with the results obtained in Ludwig \& Simner's (2013) study in which a similar linear relationship was found between lightness and three tactile sensations. They found that lighter colors were associated with smoother, softer, and rounder tactile sensations. There are several ways to look at our results. First, brightness is a polar dimension, while hue is a circular one (Marks, 1978). Considering that haptic properties also are polar, they are more likely to be naturally linked to another polar dimension rather than a circular one. Second, brightness and haptic stimuli intensity can be both viewed as prothetic perceptual continua. Prothetic perceptual continua refer to the magnitude as opposed to metathetic continua that represent quality of the sensory experience (Stevens, 1957). Both brightness and haptic 


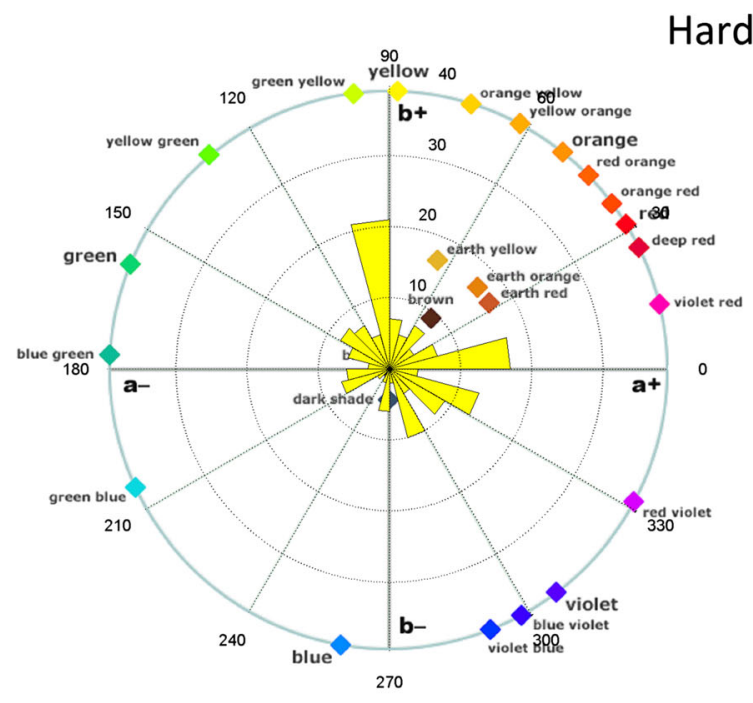

level 1
Hardness

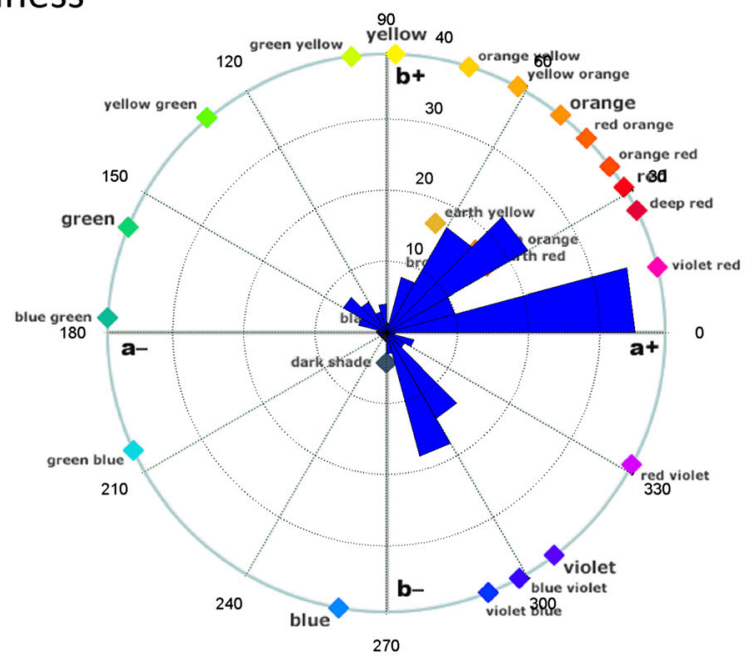

level 6

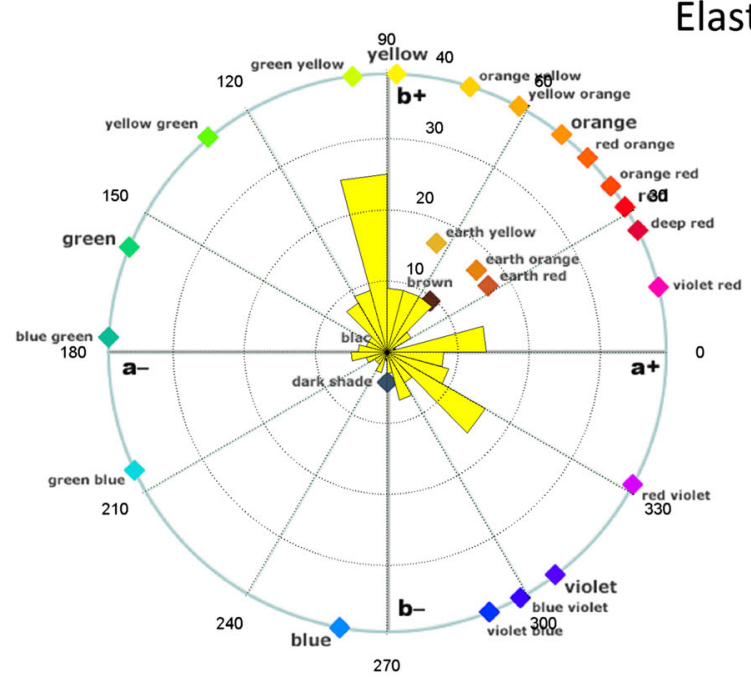

level 1

Elasticity

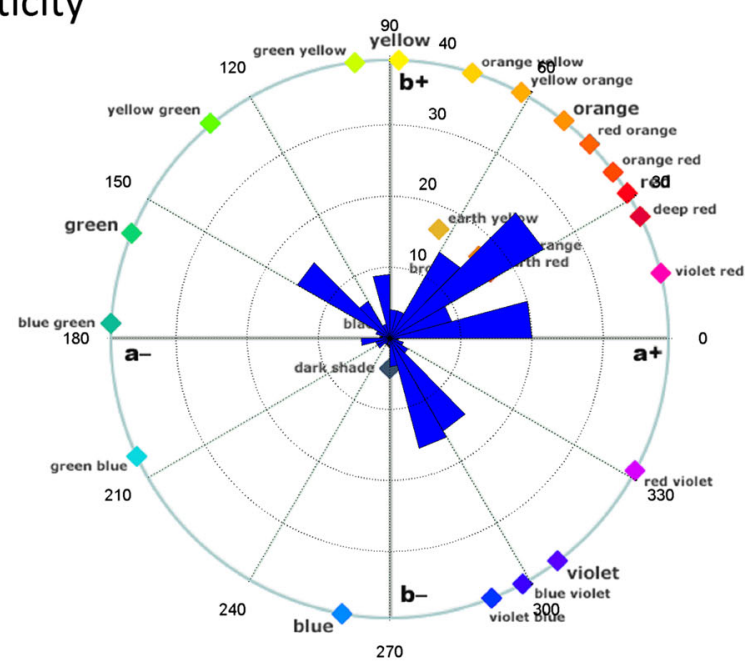

level 6

Fig. 6 Histograms of the CIEL*a*b* data points (i.e., participant responses) for level 1 and level 6 for the hardness and elasticity stimuli with an interval of 15 degrees projected onto the $\mathrm{a}^{*} \mathrm{~b} *$ plane

intensity represent a clear magnitude dimension and can be analyzed in terms of "more" or "less" of a given attribute.

Another way to look at this result would be from the standpoint of semantic hypothesis. Hard-soft and rough-smooth mainly represent potency dimension in Osgood \& Suci's (1955) analysis, but they also are related to the evaluative meaning. Considering that bright-dark scale represents the evaluative dimension, bright/soft and bright/smooth, bright/ light, bright/elastic, and bright/nonadhesive associations in our study might have occurred due to the shared positive evaluative meaning. A more distant connection also could be assumed with regard to Osgood and Suci's dimensions. Hard, rough, adhesive, inelastic, and heavy haptic stimuli were produced using larger forces (i.e., pushing on the hard surface resulted in bigger force feedback than pushing on the soft surface). These sensations could be viewed on the tense side of the tense-relaxed scale, which is representative of both the activity dimension and the negative side of the evaluative dimension. Taking into account the negative evaluative meaning of dark, dark/hard, dark/rough, dark/heavy, dark/inelastic, and dark/adhesive associations confirm the evaluative semantic mapping.

Our investigation does not allow concluding whether the found association between brightness and haptic intensity could be statistical in nature. A substantial environmental sampling would be needed to test the hypothesis that brighter stimuli in our environment are softer, smoother, lighter, more elastic, and nonadhesive. Such statistical regularities, if they exist, may be grounded in the animate/inanimate distinction between environmental stimuli. 


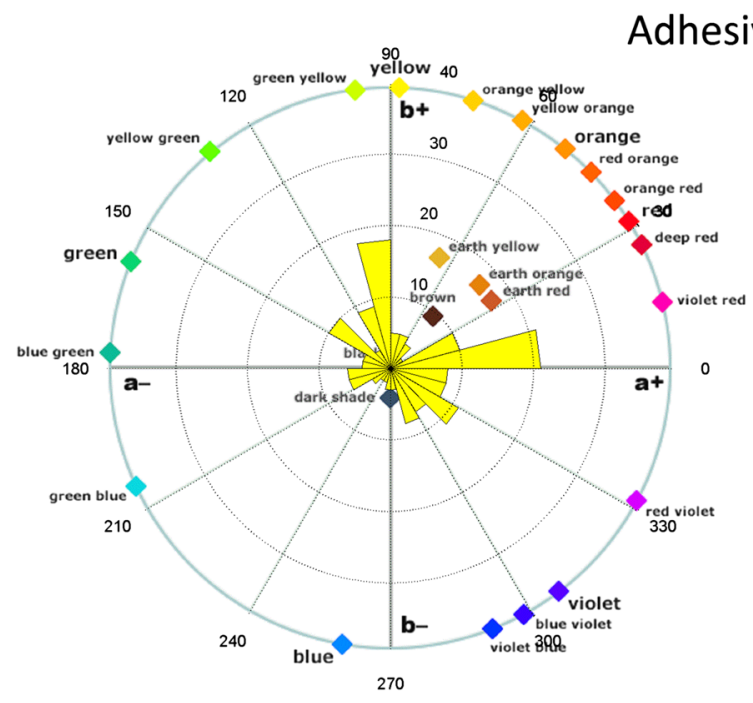

level 1

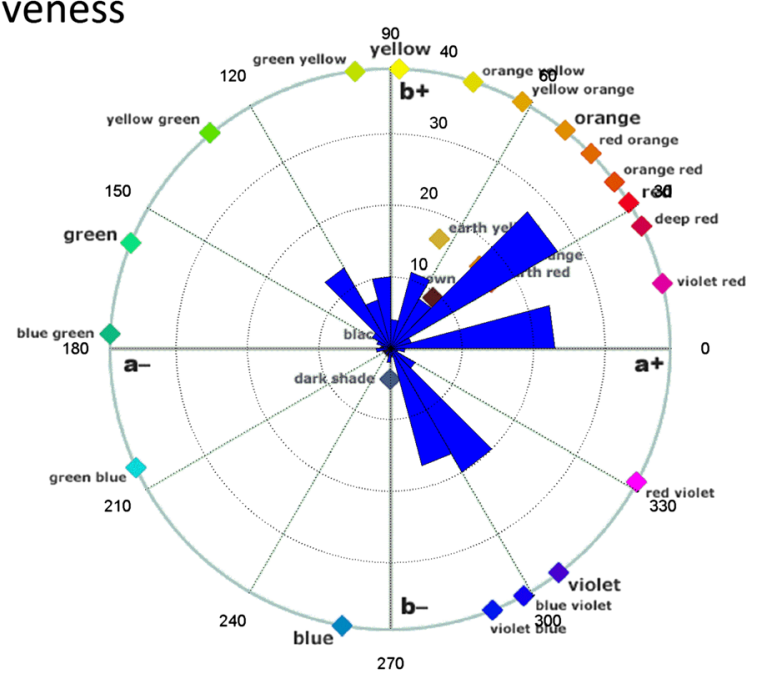

level 6

Fig. 7 Histograms of the CIEL*a*b* data points (i.e., participant responses) for level 1 and level 6 for the adhesiveness stimuli with an interval of 15 degrees projected onto the $\mathrm{a}^{*} \mathrm{~b}^{*}$ plane

Color choices for the least intense haptic stimuli were associated with the lowest degree of chroma. The highest degree of chroma, on the other hand, was characteristic to intermediate levels of haptic stimuli intensity. In other words, stimuli that were neither very rough nor very smooth, not very heavy nor very light, not very hard nor very soft, not very inelastic

Table 6 Interval-based comparison of level 1 and 6 of five haptic stimuli

\begin{tabular}{lll}
\hline & Level 1 & Level 6 \\
\hline Roughness & & \\
MAX & 15 & 30 \\
MIN & 2 & 0 \\
SD & 2.875906 & 8.742854 \\
Hardness & & \\
MAX & 21 & 35 \\
MIN & 0 & 0 \\
SD & 4.736824 & 8.666266 \\
Heaviness & & \\
MAX & 15 & 34 \\
MIN & 1 & 0 \\
SD & 4.095221 & 10.20723 \\
Elasticity & & \\
MAX & 25 & 25 \\
MIN & 1 & 0 \\
SD & 5.410253 & 6.917671 \\
Adhesiveness & & 35 \\
MAX & 25 & 0 \\
MIN & 0 & 13.27643 \\
SD & 8.229796 & \\
\hline
\end{tabular}

nor very elastic, not very adhesive, nor totally nonadhesive were linked to the most colorful color choices among all color choices. One of the peculiarities of the saturated colors is that they are generally preferred more than nonsaturated ones. Camgoz, Yener, \& Guvenc (2002) in a study on preference for foreground-background color relationship found that the participants preferred colors with maximum saturation and brightness that were available. Tangkijviwat, Rattanakasamsuk, \& Shinoda (2010) in their study on color appearance mode and color preference have shown high preference scores for color chips with high chroma and high brightness. Most recently Schloss, Strauss, \& Palmer (2013) have shown that people did not prefer saturated colors in the object context; however, they preferred saturated colors when they were presented as contextless squares of color. Considering such preference-related peculiarity of saturated colors, one way to look at our results of the match between highest degree of chroma and intermediate haptic intensity would be from perspective of haptic preference. It is possible that the found association is semantic in nature and that saturated colors that are generally preferred were matched to the haptic stimuli of intermediate intensity that were also preferred. The important aspect of such interpretation would be the definition of the haptic preference or rather the identification of what the preference could be based on. Two notions could be relevant here: haptic comfort and tactile pleasantness. Tactile pleasantness refers to the hedonic ratings of tactile sensations and has been studied before (Essick et al., 2010; Ripin \& Lazarsfeld, 1937). Haptic comfort has not been conceptualized in the literature and, in our view, pertains to a neutral experience of optimal effort and easiness of perception. While such distinction has not been made in the 
Table 7 Interval-based comparison of hue biases for level 1 and 6 responses of five haptic stimuli

\begin{tabular}{|c|c|c|c|}
\hline & Large hue biases & Medium hue biases & Small hue biases \\
\hline \multicolumn{4}{|l|}{ Level 1} \\
\hline Roughness & & & Violet red $[0,15)$ \\
\hline Hardness & & Yellow, green yellow $[90,105)$ & Violet red $[0,15)$ \\
\hline Heaviness & & & Violet red $[0,15)$ \\
\hline Elasticity & & Yellow, green yellow $[90,105)$ & Red violet $[315,330)$ \\
\hline Adhesiveness & & Violet red $[0,15)$ & Yellow, green yellow $[90,105)$ \\
\hline \multicolumn{4}{|l|}{ Level 6} \\
\hline Roughness & Violet red $[0,15)$ & $\operatorname{Red}[30,45)$ & Orange $[45,60)$, Blue violet $[300,315$ \\
\hline Hardness & Violet red $[0,15)$ & $\operatorname{Red}[30,45)$ & Orange $[45,60)$, Violet blue $[285,300$ \\
\hline Heaviness & Violet red $[0,15)$, red $[30,45)$ & Blue violet $[300,315)$ & Violet blue $[285,300)$ \\
\hline Elasticity & & Violet red $[0,15)$, red $[30,45)$ & $\begin{array}{l}\text { Yellow green }[135,150) \text {, Violet blue } \\
{[285,300), \text { Blue violet }[300,315)}\end{array}$ \\
\hline Adhesiveness & & $\begin{array}{l}\text { Violet red }[0,15), \text { red }[30,45), \\
\text { blue violet }[300,315)\end{array}$ & Violet blue $[285,300)$ \\
\hline
\end{tabular}

Numbers in the brackets indicate a degree interval in CIEL*a*b* hue plane

tactile/haptic literature before, the dissociation between pleasantness and comfort is not uncommon in other sensory experiences, thermal sensation in particular. For example, Kwon \& Parsons (2008) in their study on heat and cold indices asked participants to rate pleasantness and comfort of the temperature in the room on the separate scales. Preference also was rated separately, indicating complexity of the sensory experience that cannot be adequately conveyed by the ratings of preference, comfort, or pleasantness alone. While these experiential judgments are certainly not entirely independent, it is possible that the experience of comfort is more closely matched with "neither pleasant nor unpleasant" point on the hedonic scale rather than "very pleasant" or "pleasant." In the case of haptics, sensations of substance properties do not seem to have an inherent emotional component. For example, the weight property is detached from any emotional evaluative meaning; it is neither pleasant nor unpleasant. Whereas the weight experience can be perceptually uncomfortable as in case when the object is heavy and the interaction requires effort, this experience of perceptual discomfort still cannot be considered an emotional one. For the sensation of weight to acquire an emotional evaluative meaning of "unpleasant," the interaction would need to be prolonged and cause a muscle and joint fatigue. The same reasoning could be applied to other material properties investigated in our study. Elasticity detached from object identity is neither pleasant nor unpleasant, but inelastic objects require more effort to be stretched; adhesiveness is neither pleasant nor unpleasant, but separating from adhesive objects requires more effort. It is certainly true that in the experimental conditions participants report some ratings of pleasantness of the tactile sensations (Etzi, Spence, \& Gallace, 2014; Verrillo, Bolanowski, \& McGlone, 1999). However, when one is explicitly asked to rate the pleasantness of the experience, certain pleasantness ratings are granted. Therefore, it could be doubted that material properties detached from object identity elicit evaluative emotional judgments when the judgment is not prompted, when no extreme sensation is experienced, and when the context does not involve sensation focus (e.g., interpersonal interaction). Accordingly, if the preference for haptic experiences of certain intensity is to be assumed, it might not be based on the sense of pleasantness but rather on the experience of haptic comfort. Apart from such purely theoretical speculation, it is worth noting that the findings in the literature on hedonic ratings of tactile sensations cannot support the assumption of the preference-based match between saturated colors and haptic sensations of intermediate intensity. With regard to the tactile pleasantness, the literature has systematically shown that smooth surfaces are considered pleasant while rough surfaces are considered unpleasant (Etzi, Spence, \& Gallace, 2014; Ripin \& Lazarsfeld, 1937; Verrillo, Bolanowski, \& McGlone, 1999). It is worth noting that research on hedonic ratings of tactile sensations has traditionally focused on properties of roughness/smoothness and stiffness/softness. Nothing is known about the pleasantness of weight, elasticity, or adhesiveness as pure substance properties. Therefore, while the existing research on hedonic ratings of tactile sensations does not seem to explain our results, and we make a point against the relevance of the notion of pleasantness in the case of substance properties detached from object identity, there is a space for empirical exploration of the relationship between hedonic ratings of a wider range of tactile experiences and haptic preference.

The second notion that is relevant to the assumption of preference-based match between highly saturated colors and haptic sensations of intermediate intensity is haptic comfort. 
The highest haptic comfort, defined as optimal effort and easiness of perception, is likely to be characteristic to stimuli of intermediate intensity. Haptic stimuli of intermediate intensity in our study did not produce an intense force feedback due to the interaction with the virtual surface but rather a mild one and were easily recognizable. On the other hand, stimuli that were least intense (i.e., smoothest, softest, most elastic, lightest, most nonadhesive) were created by low-intensity vibrotactile feedback and required more perceptual effort; most intense haptic sensations were created by largest forces produced due to pressing on, pulling, and releasing of the virtual stimuli. We consider a possibility that most haptically comfortable stimuli were preferred by the participants and, therefore, were matched with most saturated stimuli that were also preferred. While current research did not measure haptic comfort, haptic preference or the saturation preference, there is some evidence in the literature that such semantic interpretation could hold true and is worth testing in the future. For example, Stevenson, Rich, \& Russell (2012) have reported the match between the odors that were more familiar and easily identifiable with more saturated colors and indicated the semantic nature of such match.

Our result on chroma and haptic intensity association contradicts the findings of the Ludwig \& Simner's (2013) study in which chroma values for the smoothest stimulus were significantly higher than for the roughest and chroma values for the softest stimulus were significantly higher than those for the hardest stimulus. This effect of chroma, however, was found by Ludwig and Simner exclusively in children (5-9 years) and adolescent (10-18 years) sample, but not in adult sample. Additionally, effect sizes for these findings were within small to medium range. Taking into consideration the effect sizes in Ludwig and Simner's study and the differences in our samples (the participants in our study were students age 18 years and older who in Ludwig and Simner's study qualify as adults), the definite conclusion on the role of saturation in cross-modal associations between color and tactile sensations calls for more empirical research.

The patterns of response distribution in color space with regard to hue were very similar for all five haptic stimuli. However, certain biases towards red, violet red, violet blue, and blue violet spectra occurred in case of stimuli with the highest intensity. Biases towards specific hues were smaller for least intense stimuli and were most notable in the yellow, green yellow, and violet red spectra. The green spectrum was the least chosen, despite the fact that unique green represents an area in perceptual space much larger than unique red, unique blue, and unique yellow (Kuehni, 2004). It should be noted that in our study participants chose colors on an HSV model, which is a cylindrical representation of the RGB space where red, green, and blue are the three primaries. All three hues arguably have equivalent areal representation (Smith, 1978). In relation to $a^{*}$ and $b^{*}$ axes, color matches occurred mostly to the right of the yellow-blue axis $\left(b^{*}\right)$ passing through the reds $(\mathrm{a}+)$. More specifically, the color choices were not exactly aligned with the $b^{*}$ axis (90 and 270 degrees) but were shifted counter clockwise, thereby ranging from approximately 150 to 300 degrees, with the largest biases within the range of 90 to 300 degrees. There was insignificant number of matches to the left of the $b^{*}$ axis, that is passing through $\mathrm{a}-$, beyond 150 degrees. It should be noted that $\mathrm{a}^{*}$ and $\mathrm{b}^{*}$ axes are not taken to have unique hues at their extremes. Unique blue and green are taken to be slightly shifted clockwise from $\mathrm{b}$ - and a- respectively, whereas unique red is taken to be shifted counter-clockwise from a+.

Considering the high similarity of such hue biases for all haptic stimuli, there is a possibility that while brightness and saturation of the color were chosen to match the sensation, the selection of the hue itself was a result of the natural preference. Research shows variability in object color preference; however, hue biases found in our study are close to those found in studies on object-independent single color preference. For example, a classical study by Eysenck (1941) found that blue was most preferred and followed by red. Camgoz, Yener, \& Guvenc (2002) found that participants preferred blue hue regardless of the background. Saito (1996) found that vivid blue was most preferred and followed by white and vivid red in a sample of Japanese participants and white and light violet in a sample of Taiwanese participants. Most recently, Fortmann-Roe (2013) has shown that blue preference is also well represented outside the experimental conditions. Fortmann-Roe analyzed more than 1 million Twitter accounts and found that blue colors were the most preferred. Some gender differences also were noted; males preferred blues more than females and females preferred magentas more than males. Males also preferred darker colors than females. As for the least preferred colors, yellow and yellowgreen systematically emerge in the literature (Eysenck, 1941; Guilford \& Smith, 1959). This also is consistent with our findings, because these hues were not selected significantly for any of the haptic stimuli with full intensity, and a small bias towards yellow, green-yellow spectrum occurred in case of the least intense stimuli, which required more haptic exploration, and could possibly represent the least comfortable and most confusing haptic sensation. It is worth noting that some cross-cultural variability in color preference has been acknowledged in the literature (Yokosawa et al., 2010), and the universality of color preference is still a matter of discussion (Taylor,Clifford, \& Franklin, 2013). The preference-based interpretation of the hue bias and saturation choice is in line with our experimental setup as per instruction, participants would first choose a hue with a certain saturation level and then move the separate brightness slider to make the final choice. If not satisfied, participants could nevertheless go back to the color wheel and pick a different hue and saturation level.

A viable alternative to the preference explanation of the hue biases is grounded in the analysis of the peculiarities of the chosen colors. The experience of color is a result of interaction 
of brightness, saturation (or chroma), and hue. Color spaces representing human color experience are not regular. The level of chroma that can be obtained depends on the hue and brightness of the color. Not all colors can reach the same levels of chroma. Connectedly, some colors are only experienced at some brightness levels. For example, we usually experience blue and green as having bright, medium, and dark shades, even if we have a tendency to consider that the best examples of "blue" or "green" are of medium brightness (Cook, Kay, \& Regier, 2005). Even when they are less saturated, these colors are still experienced as blue or green and usually are categorized as such (Cook, Kay, \& Regier, 2005). Purple also ranges across several levels of brightness. Yet, in English as well as Arabic, we tend to agree that the best example of "purple" is darker than the best example of "blue" (Berlin \& Kay, 1969). In contrast with blue or green, we do not experience yellow at all levels of brightness. Yellow is only experienced at very high levels of brightness, and a dark yellow is a brownish color. Orange also is experienced at high levels of brightness, although typically darker than yellow and darker oranges are experienced as brown. The same is true for yellow-green, where dark yellow-greens are olive or brownish types of color.

In our study, participants matched haptic stimuli with lowest level of intensity with the highest degree of brightness, lowest degree of chroma, and some bias towards yellow, green-yellow, and violet-red spectra. At high levels of brightness, colors of low chroma are close to white. The fact that hue biases were smaller in low levels of haptic intensity than in higher levels suggests that at these low levels of haptic intensity, the matched hue was not as important as in higher levels of intensity; what seemed to matter was closeness to white. Considering that yellow surfaces, specifically greenish-yellow, are the ones after white that can reach highest levels of lightness (Mollon, 2006), the hue bias towards yellow, greenyellow in case of haptic stimuli with lowest intensity could be an outcome of the brightness-based decision. In the case of the most intense haptic sensations, haptic stimuli were linked to low brightness, intermediate saturation, and most prominent hue biases in the violet red, red, and then blue-violet spectra. Smaller biases were found in the green-yellow and yellow spectra. Colors that range from green-yellow to violet-red and then blue-violet at low brightness levels and low to intermediate saturation are all brownish (see MacEvoy, 2005, for mapping of a larger number of pigments in CIEL*a*b*). Given this relationship, dark brownish colors were the most frequent match for the most intense haptic stimuli. Despite this, it should be noted that we did not find clear level-based clustering of participant color choices, but rather showed that areas in the three-dimensional color space representing participant responses become more distinctive as the intensity of the compared stimuli becomes more different. We consider this initial finding a starting point for a larger scale study on the relationship between haptic stimuli and color.
The findings of the role of hue and color as a whole in cross-modal mapping between tactile sensations have been inconclusive in other studies. Ludwig \& Simner (2013) have indicated a tendency to associate certain tactile sensations to specific colors (incidentally rough to dark browns). However, the colors were identified on the basis of the two authors' categorization of the participants' responses using 11 color terms proposed by Berlin \& Kay (1969). We believe that introducing color categories as a tool to measure associations of haptic sensations to color sensations may have potentially obscured the results. Ultimately, this method would show associations between haptic sensations and color lexical categories, rather than hue and color.

There is also a remote possibility that the hue biases found in our study are an example of the behavioral asymmetries. Such asymmetries with a bias towards the right side were shown on a number of behaviors, including the higher probability of turning to the right when kissing (Barrett, Greenwood, \& McCullagh, 2006), the tendency to turn right in a T-maze (Scharine and McBeath, 2002), and others. An example of the lateral bias that is most relevant to our case, because it also depicts bias in choice from the display, is a tendency to choose theatre seats from the right side of the seating chart (Karev, 2000), which was confirmed by actual seating behavior (Harms, Reese, and Elias, 2014). We consider the possibility of the lateral bias interpretation due to the fact that green-yellow, red, violet, and blue-violet spectra that represent hue biases across all stimuli were situated on the right side of the HSV color wheel used in the study. Such interpretation, however, as well as the existence of the lateral bias in color preference requires an empirical investigation.

Overall, our study revealed cross-modal associations between color properties and haptic sensations, including sensations that have never been researched in such context (i.e., elasticity, adhesiveness). We have also introduced haptically rendered virtual stimuli to the field of cross-modal mapping research. The consistency between our findings and the findings pertaining to interaction with real objects suggests that virtual stimuli present a good alternative to real stimuli with an added benefit of avoiding interference of irrelevant stimuli properties. Our results find application in the field of haptic color rendering. The found systematic associations between haptic intensity and chroma, and haptic intensity and brightness suggest an ecologically salient haptic color rendering strategy.

Future research might look into the use of different crossmodal matching paradigms to replicate our findings as well as link them to other cross-modal associations with color. Some of the issues that the future research on haptics in the present context might consider are the effect of the vibrotactile adaptation and the differences between vibrotactile feedback provided to the hand of the participant who behaves as a passive receiver of such feedback and the vibrotactile feedback that is 
a result of the participant's active investigation of the virtual stimulus. The latter case was the case of the present study, because haptic display interaction by its nature requires such active effort on the side of the participant; however, we consider the possibility that the participant responses were determined by a relatively complex sensation resulting from the active interaction with the device as opposed to a more simple sensation of the five substance properties.

\section{References}

Albertazzi, L., \& Da Pos, O. (2012). The Hue of Shapes. Journal of Experimental Psychology: Human Perception and Performance, 39(1), 37-47.

Amedi, A., Jacobson, G., Hendler, T., Malach, R., \& Zohary, E. (2002). Convergence of visual and tactile shape processing in the human lateral occipital complex. Cerebral Cortex, 12(11), 1202-1212.

Barrett, D., Greenwood, J. G., \& McCullagh, J. F. (2006). Kissing laterality and handedness. Laterality, 11, 573-579.

Berlin, B., \& Kay, P. (1969). Basic Colour Terms: Their Universality and Evolution. Berkeley: University of California Press.

Bicchi, A., Buss, M., Ernst, M. O., \& Peer, A. (Eds.). (2008). The sense of touch and its rendering. Progress in haptics research: Vol. 45. Springer Tracts in Advanced Robotics. Berlin: Springer.

Camgoz, N., Yener, C., \& Guvenc, D. (2002). Effects of hue, saturation, and brightness on preference. Color Research and Application, 27(3), 199-207.

Cook, R., Kay, P., \& Regier, T. (2005). The World Color Survey database: History and use. In H. Cohen \& C. Lefebvre (Eds.), Handbook of categorization in the cognitive sciences (pp. 224-242). Amsterdam: Elsevier.

Culbertson, H., Unwin, J., \& Kuchenbecker, K. J. (2014). Modeling and Rendering Realistic Textures from Unconstrained Tool-Surface Interactions. IEEE Transactions on Haptics, 7(3), 381-393.

Deroy, O., \& Spence, C. (2013a). Why we are not all synesthetes (not even weakly so). Psychonomic Bulletin \& Review, 20, 1-22.

Deroy, O., \& Spence, C. (2013b). Are we all born synaesthetic? Examining the neonatal synaesthesia hypothesis. Neuroscience and Biobehavioral Reviews, 37, 1240-1253.

Drewing, K., Ernst, M. O., Lederman, S. J., \& Klatzky, R. (2004). Roughness and spatial density judgments on visual and haptic textures using virtual reality. In M. Buss \& M. Fritschi (Eds.), Proceedings of the 4th International Conference EuroHaptics 2004 (pp. 203-206). Munich: Herbert Hieronymus.

Essick, G. K., McGlone, F., Dancer, C., Fabricant, D., Ragin, Y., Phillips, N., ... Guest, S. (2010). Quantitative assessment of pleasant touch. Neuroscience and Biobehavioral Reviews, 34, 192-203.

Etzi, R., Spence, C., \& Gallace, A. (2014). Textures that we like to touch: An experimental study of aesthetic preferences for tactile stimuli. Consciousness and Cognition, 29, 178-188.

Eysenck, H. J. (1941). A critical and experimental study of color preferences. American Journal of Psychology, 54, 385-394.

Fairchild, M. D. (2013). Color Appearance Models (3rd Edition). John Wiley \& Sons, Ltd.

Fortmann-Roe, S. (2013). Effects of Hue, Saturation, and Brightness on Color Preference in Social Networks: Gender-Based Color Preference on the Social Networking Site Twitter. Color Research and Application, 38(3), 196-202.
Gilbert, A. N., Martin, R., \& Kemp, S. E. (1996). Cross-modal correspondence between vision and olfaction: The color of smells. American Journal of Psychology, 109, 335-351.

Glicksohn, A., \& Cohen, A. (2013). The role of cross-modal associations in statistical learning. Psychonomic Bulletin \& Review, 20, 11611169

Guilford, J. P., \& Smith, P. C. (1959). A system of colour preferences. American Journal of Psychology, 72, 487-502.

Harms, V., Reese, M., \& Elias, L. J. (2014). Lateral bias in theatre-seat choice. Laterality, 19(1), 1-11.

Hubbard, T. L. (1996). Synesthesia-like Mappings of Lightness, Pitch, and Melodic Interval. The American Journal of Psychology, 109(2), 219-238.

Jones, G. M., Bokinsky, A., Tretter, T., \& Negishi, A. (2005). A Comparison of Learning with Haptic and Visual Modalities. Haptics-e, 3(6). Retrieved from http://www.haptics-e.org/Vol_03/ he-v3n6.pdf

Karev, G. B. (2000). Cinema seating in right, mixed, and left handers. Cortex, 36, 747-752.

Kim, Y.-J. (2013). Can Eyes Smell? Cross-Modal Correspondences Between Color Hue-Tone and Fragrance Family. Color Research and Application, 38(2), 139-156.

Kitamura, E., Miyashita, K., Ozawa, K., Omata, M., \& Imamiya, A. (2006). Cross-Modality Between Haptic and Auditory Roughness with a Force Feedback Device. Journal of Robotics and Mechatronics, 18(4), 450-457.

Kuehni, R. G. (2004). Variability in Unique Hue Selection: A Surprising Phenomenon. Color Research and Application, 29(2), 158-162.

Kwon, J. Y., \& Parsons, K. (2008). Heat and Cold Stress Indices for People Exposed to Our Changing Climate. In S.-D. Yoo (Ed.), EKC2008 Proceedings of the EU-Korea Conference on Science and Technology (pp. 467-475). Heidelburg: Springer-Verlag.

Lindbloom, B. J. (2014). Retrieved from http://brucelindbloom.com/

Ludwig, V. U., \& Simner, J. (2013). What colour does that feel? Tactilevisual mapping and the development of cross-modality. Cortex, 49(4), 1089-99.

MacEvoy, B. (2009). Retrieved from http://www.handprint.com/HP/ WCL/vismixmap.html\#CIELAB

MacEvoy, B. (2005). Retrieved from http://www.handprint.com/HP/ WCL/labwheel.html

Marks, L. (1978). The unity of the senses: Interrelations among the modalities. New York: Academic Press.

Martino, G., \& Marks, L. E. (1999a, November). Cross-modal interaction in vision and touch. Poster session presented at the 40th Annual Meeting of the Psychonomics Society, Los Angeles.

Martino, G., \& Marks, L. E. (1999b). Perceptual and linguistic interactions in speeded classification: Tests of the semantic coding hypothesis. Perception, 28, 903-923.

Martino, G., \& Marks, L. E. (2001). Synesthesia: Strong and weak. Current Directions in Psychological Science, 10, 61-65.

Mitchel, A. D., \& Weiss, D. J. (2011). Learning Across Senses: CrossModal Effects in Multisensory Statistical Learning. Journal of Experimental Psychology: Learning, Memory, and Cognition, 37(5), 1081-1091.

Mollon, J. (2006). The Verriest Lecture, Lyon, July 2005. Visual Neuroscience, 23, 297-309.

Osgood, C. E. (1952). The nature and measurement of meaning. Psychological Bulletin, 49(3), 197-237.

Osgood, C. E., \& Suci, G. J. (1955). Factor analysis of meaning. Journal of Experimental Psychology, 50(5), 325-338.

Osgood, C. E., Suci, G. J., \& Tannenbaum, P. H. (1957). The measurement of meaning. Urbana: University of Illinois Press.

Palmer, S. E., Schloss, K. B., Xu, Z., \& Prado-León, L. R. (2013). Musiccolor associations are mediated by emotion. PNAS, 110(22), 88368841 . 
Parise, C. V., Knorre, K., \& Ernst, M. O. (2014). Natural auditory scene statistics shapes human spatial hearing. PNAS, 111(16), 6104-6108.

Parise, C. V., \& Spence, C. (2012). Audiovisual crossmodal correspondences and sound symbolism: A study using the implicit association test. Experimental Brain Research, 220(3-4), 1-15.

Ripin, R., \& Lazarsfeld, P. F. (1937). The tactile-kinaesthetic perception of fabrics with emphasis on their relative pleasantness. Journal of Applied Psychology, 21, 198-224.

Saito, M. (1996). Comparative studies on color preference in Japan and other Asian regions, with special emphasis on the preference for white. Color Research and Application, 21(1), 35-49.

Scharine, A. A., \& McBeath, M. K. (2002). Right-handers and Americans favor turning to the right. Human Factors, 44, 248-256.

Schifferstein, H. N. J., \& Tanudjaja, I. (2004). Visualising fragrances through colours: The mediating role of emotions. Perception, 33(10), 1249-1266.

Schloss, K. B., Strauss, E. D., \& Palmer, S. E. (2013). Object color preferences. Color Research and Application, 38(6), 393-411.

Smith, A. R. (1978). Color Gamut Transform Pairs. ACM Siggraph Computer Graphics, 12(3), 12-19.

Spector, F., \& Maurer, D. (2009). Synesthesia: A new approach to understanding the development of perception. Developmental Psychology, 45, 175-189.

Spector, F., \& Maurer, D. (2011). The Colors of the Alphabet: NaturallyBiased Associations Between Shape and Color. Journal of Experimental Psychology: Human Perception and Performance, $37(2), 484-495$.

Spence, C. (2011). Crossmodal correspondences: A tutorial review. Attention, Perception, and Psychophysics, 73, 971-995.

Spence, C., \& Deroy, O. (2012). Crossmodal correspondences: Innate or learned? i-Perception, 3, 316-318.
Spence, C., Levitan, C. A., Shankar, M. U., \& Zampini, M. (2010). Does food color influence taste and flavor perception in humans? Chemosensory Perception, 3(1), 68-84.

Stevens, S. S. (1957). On the psychophysical law. Psychological Review, 64, 153-181.

Stevenson, R. J., Rich, A., \& Russell, A. (2012). The nature and origin of cross-modal associations to odours. Perception, 41, 606-619.

Tangkijviwat, U., Rattanakasamsuk, K., \& Shinoda, H. (2010). Color Preference Affected by Mode of Color Appearance. Color Research and Application, 35(1), 50-63.

Taylor, C., Clifford, A., \& Franklin, A. (2013). Color Preferences Are Not Universal. Journal of Experimental Psychology: General, 142(4), 1015-1027.

Unger, B., Hollis, R., \& Klatzky, R. (2011). Roughness Perception in Virtual Textures. IEEE Transactions on Haptics, 4(2), 122-133.

Verrillo, R. T., Bolanowski, S. J., \& McGlone, F. P. (1999). Subjective magnitude of tactile roughness. Somatosensory and Motor Research, 16, 352-360.

Yokosawa, K., Yano, N., Schloss, K. B., Prado-Leòn, L. R., \& Palmer, S. E. (2010). Cross-Cultural Studies of Color Preferences: US, Japan, and Mexico. Journal of Vision, 10(7), 408.

Walsh, V. (2003). A theory of magnitude: common cortical metrics of time, space and quantity. Trends in Cognitive Sciences, 7(11), 483488.

Ward, J., Huckstep, B., \& Tsakanikos, E. (2006). Sound-colour synaesthesia: To what extent does it use cross-modal mechanisms common to us all? Cortex, 42, 264-280.

Woods, A. T., Spence, C., Butcher, N., \& Deroy, O. (2013). Fast lemons and sour boulders: Testing crossmodal correspondences using an internet-based testing methodology. i-Perception, 4(6), 365. 